\title{
Mechanisms through which a small protein and lipid preload improves glucose tolerance
}

\author{
Domenico Tricò $^{1,2} \cdot$ Simona Baldi $^{1} \cdot$ Alberto Tulipani $^{1} \cdot$ Silvia Frascerra $^{1}$. \\ Maria Paula Macedo ${ }^{3,4}$ - Andrea Mari $^{5}$ - Ele Ferrannini ${ }^{1,6}$ - Andrea Natali ${ }^{1}$
}

Received: 22 April 2015 / Accepted: 7 July 2015 / Published online: 30 July 2015

(C) Springer-Verlag Berlin Heidelberg 2015

\begin{abstract}
Aims/hypothesis Small protein or lipid preloads are able to improve glucose tolerance to a different extent and through different and poorly defined mechanisms. We aimed at quantifying the effect of a mixed protein and lipid preload and at evaluating the underlying mechanisms.

Methods Volunteers with normal (NGT, $n=12)$ or impaired (IGT, $n=13$ ) glucose tolerance and patients with type 2 diabetes $(n=10)$ underwent two OGTTs coupled to the double glucose tracer protocol, preceded by either $50 \mathrm{~g}$ of parmesan cheese, a boiled egg and $300 \mathrm{ml}$ of water, or $500 \mathrm{ml}$ of water. We measured plasma glucose, insulin, C-peptide, glucagon-like peptide-1 (GLP-1), glucose-dependent insulinotropic polypeptide (GIP), pancreatic polypeptide (PP), NEFA and glucose tracers, and calculated glucose fluxes, beta cell function variables, insulin sensitivity and clearance.
\end{abstract}

Electronic supplementary material The online version of this article (doi:10.1007/s00125-015-3710-9) contains peer-reviewed but unedited supplementary material, which is available to authorised users.

Domenico Tricò

domenico.trico@for.unipi.it

1 Department of Clinical and Experimental Medicine, University of Pisa, Via Roma 57, Pisa 56100, Italy

2 Institute of Life Sciences, Scuola Superiore Sant'Anna, Pisa, Italy

3 CEDOC, NOVA Medical School/Faculdade de Ciencias Medicas (NMS/FCM), Universidade Nova de Lisboa, Lisboa, Portugal

4 APDP-Diabetes Portugal, Education and Research Centre (APDP-ERC), Lisboa, Portugal

5 National Research Council, Institute of Neuroscience, Padua, Italy

6 National Research Council, Institute of Clinical Physiology, Pisa, Italy
Results After the nutrient preload, the OGTT-induced rise of plasma glucose was lower than after water alone in each study group. This reduction-more pronounced across classes of glucose tolerance (NGT $-32 \%$, IGT $-37 \%$, type 2 diabetes $-49 \% ; p<0.002$ ) - was the result of different combinations of slower exogenous glucose rate of appearance, improved beta cell function and reduced insulin clearance, in this order of relevance, which were associated with an only mild stimulation of GIP and GLP-1.

Conclusions/interpretation After a non-glucidic nutrient preload, glucose tolerance improved in proportion to the degree of its baseline deterioration through mechanisms that appear particularly effective in type 2 diabetes. Exploiting the physiological responses to nutrient ingestion might reveal, at least in the first stages of the diabetic disease, a potent tool to improve daily life glycaemic control.

Trial registration: ClinicalTrials.gov NCT02342834

Funding: This work was supported by grants from the University of Pisa (Fondi di Ateneo) and by FCT grant (PIC/IC/ 82956/2007).

Keywords Beta cell function - Glucose absorption - Glucose intolerance $\cdot$ Hepatic glucose production $\cdot$ Insulin secretion in vivo $\cdot$ Insulin sensitivity $\cdot$ Lipid $\cdot$ Protein $\cdot$ Type 2 diabetes

$\begin{array}{ll}\text { Abbreviations } \\ \text { EGP } & \text { Endogenous glucose production } \\ \text { FPG } & \text { Fasting plasma glucose } \\ \text { GCl } & \text { Glucose clearance } \\ \text { GIP } & \text { Glucose-dependent insulinotropic polypeptide } \\ \text { GLP-1 } & \text { Glucagon-like peptide-1 } \\ \text { iAUC } & \text { Incremental AUC } \\ \text { IGT } & \text { Impaired glucose tolerance }\end{array}$


ISR Insulin secretion rate

ISR@FPG Insulin secretion rate at fasting plasma glucose concentration

NGT Normal glucose tolerance

PP Pancreatic polypeptide

$\mathrm{RaO} \quad$ Rate of appearance of oral glucose

\section{Introduction}

Experimental [1,2] and clinical data [3, 4] indicate that oral carbohydrates tolerance can be influenced by the ingestion of protein and lipid nutrients through the activation of neural reflexes, hormonal responses and gradients of plasma substrates that modulate gastric emptying, insulin secretion and insulin clearance [5-7].

The size of these effects is influenced by a number of factors, including the specific nutrient chemical $[8,9]$ and physical (e.g. solid vs liquid) $[10,11]$ characteristics, the timing (preload vs co-ingestion) $[1,2]$ and the individual glucose tolerance status [12]. In healthy participants, the ingestion of fat together with carbohydrate attenuates the rise in blood glucose [13-16] mainly by slowing down gastric emptying [15-17], with minor effects on insulin secretion [13, 15]. In patients with type 2 diabetes, fat co-ingested with carbohydrate has been shown to enhance the plasma insulin response [18] and delay gastric emptying [1]; however, this has a limited impact on plasma glucose excursions $[1,13]$ possibly due to a concomitant negative effect of plasma fat substrates on insulin sensitivity [19]. Protein co-ingested with carbohydrate exerts a hypoglycaemic effect in both normal $[6,12,14,20]$ and diabetic participants $[2,12,21]$ through the potentiation of insulin secretion $[2,12,14,21]$, the reduction of hepatic insulin clearance [6] and a minor delay of gastric emptying [2, 20]. In quantitative terms, protein ingestion has been shown to improve glucose tolerance to a threefold greater extent than fat in healthy participants [14]. The action of nutrients also depends on the timing and presumably on the metabolic and hormonal consequences of their arrival into the intestine as well as their digestion and absorption [16, 22, 23]. For instance, a small oil load was able to attenuate the postprandial rise of plasma glucose when ingested $30 \mathrm{~min}$ before the meal, while it had only a minor effect when mixed with carbohydrates [1]. Similarly, the ingestion of a small amount of whey protein was able to blunt the glycaemic excursion to a greater extent when given before rather than mixed with a highcarbohydrate meal [2]. Although the effects of protein and fat on glucose tolerance are expected to be synergistic, being the involved mechanisms partially different, the available evidence does not fully support this hypothesis [24], possibly because the nutrients were co-ingested with glucose and not given as a preload.
We designed this study to measure the size of the effect on glucose tolerance of a small mixed protein and lipid meal given as a preload and to analyse the mechanisms involved by accurately evaluating beta cell function, insulin sensitivity, insulin clearance and glucose kinetics (oral absorption, endogenous production, rate of utilisation), together with glucagon and gut hormone plasma concentrations. Since each and all the mechanisms activated by nutrients ingestion might be affected by the presence of diabetes, we studied individuals with different degrees of glucose tolerance impairment.

\section{Methods}

Participants Thirty-five volunteers were enrolled. The inclusion criteria were age $18-65$ years, BMI $18-35 \mathrm{~kg} / \mathrm{m}^{2}$, normal or impaired fasting plasma glucose (FPG) or newly diagnosed diet-controlled type 2 diabetes. None of these volunteers was on drug treatment or had diseases other than diabetes that could potentially interfere with carbohydrate absorption and metabolism. According to the standard diagnostic criteria [25], the volunteers were divided into three groups on the basis of the $2 \mathrm{~h}$ plasma glucose concentration achieved during the control OGTT: those with normal glucose tolerance (NGT), those with impaired glucose tolerance (IGT) and those with type 2 diabetes. The study was approved by the institutional Ethics Committee and all participants provided written consent to participate.

Study design This was an open, randomised clinical trial. On 2 days, separated by a period from 2-4 weeks, at 08:00 hours after an overnight fast (12 h), participants were admitted to our Clinical Research Unit and underwent a standard 75 g OGTT. All volunteers lay in bed in semi-upright sitting position with the head of the bed at approximately $45^{\circ}$. A 20 -gauge polyethylene cannula was inserted into an antecubital vein for the infusion of all test substances. A second catheter was inserted retrogradely into an ipsilateral wrist vein for blood sampling, and the hand was kept wrapped in a heated blanket to achieve arterialisation of venous blood. Thirty minutes before glucose ingestion (time $-30 \mathrm{~min}$ ), the volunteers were randomised by a coin toss to a preload of either $500 \mathrm{ml}$ of water (water) or a small, virtually glucose-free mixed meal, consisting of $50 \mathrm{~g}$ of parmesan cheese and one small-size boiled egg ( $23 \mathrm{~g}$ protein, $17 \mathrm{~g}$ fat and $2 \mathrm{~g}$ carbohydrate, to a total of $\sim 1,000 \mathrm{~kJ}$ ), followed by $300 \mathrm{ml}$ of water (nutrients). The preloads were consumed between -30 and -25 min and had the same volume, differing in the amount of water ingested, to exclude the influence of the mechanical dilatation of the stomach on gastric emptying and parasympathetic activation $[26,27]$. Thirty volunteers (ten with NGT, thirteen IGT and seven type 2 diabetes) gave their consent to undergo a standard double tracer protocol [28]. Their oral glucose drink 
consisted of $147 \mathrm{ml}$ of $50 \%$ glucose solution (wt/vol.) enriched with $1.5 \mathrm{~g}$ of $\left[\mathrm{U}_{-}{ }^{13} \mathrm{C}\right]$ glucose (Spectra 2000, Rome, Italy) in order to trace oral glucose absorption. Furthermore, a primed $(28 \mu \mathrm{mol} / \mathrm{kg})$-continuous $\left(0.28 \mu \mathrm{mol} \mathrm{min}{ }^{-1} \mathrm{~kg}^{-1}\right)$ infusion of 6,6- $\left[{ }^{2} \mathrm{H}_{2}\right]$ glucose (Spectra 2000) was started at time $-150 \mathrm{~min}$ through the antecubital vein catheter and continued until the end of the study to estimate endogenous glucose production (EGP) and the rate of disappearance of plasma glucose. The volunteers who denied consent to tracers received a $0.9 \%$ saline infusion according to the same format of the double tracer protocol. Timed arterialised blood samples were collected during the test to measure plasma glucose, insulin, C-peptide, glucagon-like peptide-1 (GLP-1), glucosedependent insulinotropic polypeptide (GIP), glucagon, NEFA, pancreatic polypeptide (PP) and glucose tracer enrichments.

Analytical procedures Plasma glucose was measured immediately by the glucose-oxidase technique (Beckman Glucose Analyzer II, Beckman Instruments, Fullerton, CA, USA). Insulin and C-peptide measurements were performed by electrochemiluminescence on a COBAS e411 instrument (Roche, Indianapolis, IN, USA). Plasma GLP-1, GIP and glucagon were assessed using a Multiplex technique (Biorad Laboratories, Hercules, CA, USA). 6,6- $\left[{ }^{2} \mathrm{H}_{2}\right]$ glucose and $\left[\mathrm{U}-{ }^{13} \mathrm{C}\right]$ glucose were measured by GC-MS as previously described [28]. Plasma PP concentrations were measured in NGT and IGT groups by ELISA (Biorad Laboratories). Plasma NEFA were assayed by standard spectrophotometric methods on a Synchron Clinical System CX4 (Beckman Instruments).

Mathematical modelling EGP, rate of appearance of oral glucose $(\mathrm{RaO})$ and plasma glucose clearance $(\mathrm{GCl})$ were assessed from the time course of the plasma tracer/tracee ratio of 6,6- $\left[{ }^{2} \mathrm{H}_{2}\right]$ glucose and $\left[\mathrm{U}^{-13} \mathrm{C}\right]$ glucose [29]. Insulin secretion rate (ISR) was estimated from C-peptide deconvolution [30]. Beta cell function variables were calculated by modelling insulin secretion and glucose concentration, as illustrated in detail previously [31-34]. Briefly, this model describes insulin secretion as the sum of three components. The first represents the dependence of insulin secretion on absolute glucose concentration and is characterised by a quasi-linear doseresponse function relating the two variables. This function is described by the slope of the line within the observed glucose range, denoted as beta cell glucose sensitivity, and by the ISR at FPG (ISR@FPG). The individual dose-response function is modulated by a time-dependent factor termed potentiation, which accounts for all the physiological processes that acutely modify insulin secretion (antecedent hyperglycaemia, gastrointestinal hormones, neurotransmitters, non-glucose substrates). The third component of insulin secretion, termed beta cell rate sensitivity, represents the dependence of insulin secretion on the rate of increase of plasma glucose concentration and therefore reflects early insulin release. The incremental area under time-concentration curves (iAUC) were calculated by the trapezoidal rule between -30 and $120 \mathrm{~min}$ with the fasting $(-30 \mathrm{~min})$ concentration of each variable used as baseline. Insulin clearance was estimated by calculating the ratio between insulin secretion iAUC and plasma insulin iAUC. Insulin sensitivity was estimated as the change between basal and 60-120 min mean $\mathrm{GCl}$ divided by the plasma insulin concentration gradient over the same time intervals.

Statistical analysis Data are given as means \pm SEM. The changes induced by our experimental manoeuvre (water vs nutrients) were evaluated by using either the two-way ANOVA for repeated measures or by the paired $t$ test for normally distributed mean values and by Wilcoxon-MannWhitney test for non-normally distributed variables. ANOVA, by allowing a more robust detection of trends in the whole study population without the limitation of the small sample size of each group, was used when the groups were found to have a homogeneous response ( $F$ test on the grouping variable non-statistically significant at the analysis between participants), while the paired comparisons were used to ascertain the size and the statistical significance of the changes within each of the three study groups and to highlight the heterogeneity of the response among groups. Standard linear multiple regression models were used to assess the determinants of glucose iAUC changes between the two studies. Statistical analyses were performed using JMP 9.0. (SAS Institute, Cary, NC, 1989-2007). A value of $p \leq 0.05$ was considered statistically significant.

\section{Results}

Study population The studies were well tolerated and none of the volunteers reported any gastrointestinal symptoms

Table 1 Clinical characteristics

\begin{tabular}{llll}
\hline & NGT $(n=12)$ & IGT $(n=13)$ & T2D $(n=10)$ \\
\hline Age (years) & $29 \pm 11$ & $47 \pm 17^{*}$ & $55 \pm 7^{\dagger}$ \\
Sex (male/female) & $6 / 6$ & $7 / 6$ & $6 / 4$ \\
Height $(\mathrm{cm})$ & $173 \pm 8$ & $168 \pm 10$ & $170 \pm 7$ \\
Weight $(\mathrm{kg})$ & $75.6 \pm 14.8$ & $73.8 \pm 14.2$ & $82.4 \pm 12.7$ \\
BMI $\left(\mathrm{kg} \mathrm{m}^{-2}\right)$ & $25.2 \pm 4.7$ & $26.3 \pm 5.9$ & $28.7 \pm 4.7$ \\
HbA $_{1 \mathrm{c}}(\%[\mathrm{mmol} / \mathrm{mol}])$ & $5.3 \pm 0.05[34]$ & $5.7 \pm 0.11[39]$ & $6.8 \pm 0.14[51]$ \\
FPG (mmol/l) & $5.1 \pm 0.1$ & $5.9 \pm 0.3$ & $7.0 \pm 0.4^{\dagger \dagger}$ \\
FPI (pmol/l) & $52.4 \pm 10.1$ & $56.6 \pm 7.3$ & $84.0 \pm 14.9$ \\
\hline
\end{tabular}

Data are means \pm SD

${ }^{*} p<0.05$ NGT vs IGT; ${ }^{\dagger} p<0.05$ NGT vs T2D; ${ }^{\star} p<0.05$ IGT vs T2D

FPI, fasting plasma insulin; T2D, type 2 diabetes 
(i.e. nausea or vomiting). The clinical characteristics of the study population are shown in Table 1. Participants with IGT and type 2 diabetes were older than those with NGT. In addition, type 2 diabetic patients had higher FPG and $\mathrm{HbA}_{1 \mathrm{c}}$ compared with those with NGT and IGT. The BMI showed a trend to increase across classes of glucose tolerance, but the difference was not statistically significant.

Plasma glucose, insulin secretion, sensitivity and clearance FPG, insulin levels and ISR were not statistically different between the two study days within each study group (Fig. 1). Before glucose ingestion ( $-30 \mathrm{~min}$ to $0 \mathrm{~min}$ ), the nutrient preload did not induce changes in plasma glucose concentration while it caused a small, though significant, increase in both plasma insulin concentrations and ISR in each group. The ingestion of water neither affected plasma glucose nor plasma insulin. Following glucose ingestion (0-120 min), the rise in plasma glucose concentration was significantly lower after the nutrient preload than after water ingestion in each group $(p<0.002$ for all). The size of this effect was progressively greater across the three classes of glucose tolerance both in absolute terms (Fig. 1) and percentage-wise (NGT $-32 \%$, IGT $-37 \%$, type 2 diabetes $-49 \%$ ). The insulin rise was slightly anticipated by the nutrient preload in all study groups while during the second hour of the test insulin secretion paralleled the plasma glucose profiles in NGT and reached similar plateaus in participants with IGT and type 2 diabetes, despite the marked differences in plasma glucose values. Although the reduction in insulin clearance failed to reach full statistical significance in the NGT group $(p=0.07$; Table 2), the ANOVA indicated that it was slightly decreased $(\sim 10 \%)$ by the nutrient preload in all study groups $(p<0.001)$ with neither effect of the glucose tolerance status nor of the interaction. Insulin sensitivity was reduced by $82 \%$ in type 2 diabetic individuals and by $55 \%$ in individuals with IGT compared with NGT and was unaffected by the nutrient preload.

Beta cell function modelling variables The early secretion (rate sensitivity) and the slope of the glucose-insulin secretion dose-response curve (glucose sensitivity) measured in the water OGTT displayed a progressive decline across glucose tolerance classes, while potentiation was similar (Table 2). In
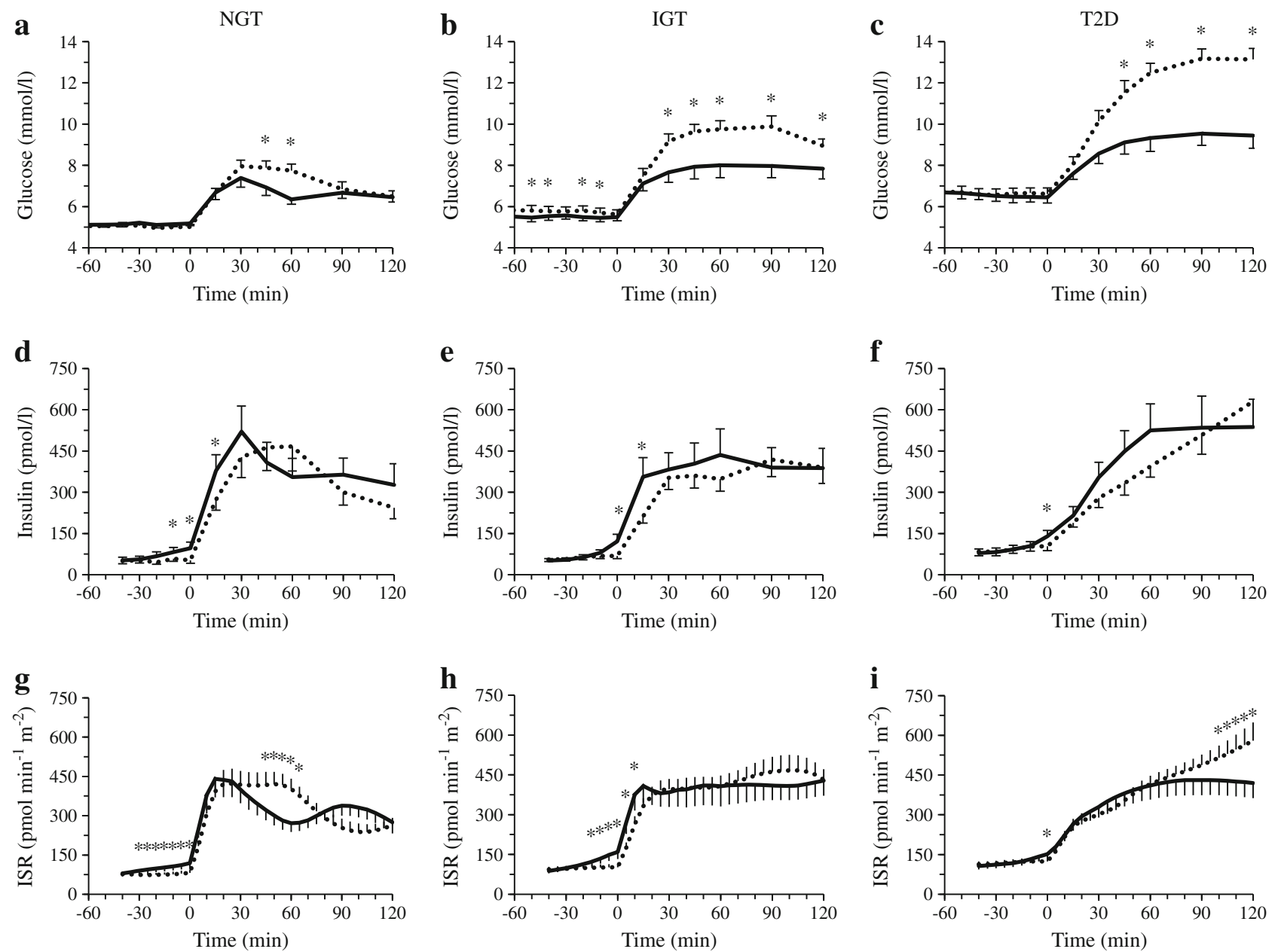

Fig. 1 Plasma glucose (a-c), plasma insulin (d-f) and ISR (g-i) during two $75 \mathrm{~g}$ OGTTs preceded at -30 min by a preload of either water (dotted lines) or nutrients + water (black line) in NGT $(\mathbf{a}, \mathbf{d}, \mathbf{g})$, IGT $(\mathbf{b}, \mathbf{e}, \mathbf{h})$ and type 2 diabetes (T2D; $\mathbf{c}, \mathbf{f}, \mathbf{i})$ groups. ${ }^{*} p<0.05$ water vs nutrients 
Table 2 Metabolic and hormonal variables

\begin{tabular}{|c|c|c|c|c|c|c|c|c|c|}
\hline & \multicolumn{3}{|l|}{ NGT } & \multicolumn{3}{|l|}{ IGT } & \multicolumn{3}{|l|}{$\mathrm{T} 2 \mathrm{D}$} \\
\hline & Water & Nutrients & $p$ & Water & Nutrients & $p$ & Water & Nutrients & $p$ \\
\hline Plasma glucose iAUC (mmol/l) & $251 \pm 20$ & $171 \pm 28$ & 0.003 & $412 \pm 26$ & $260 \pm 38$ & 0.0003 & $576 \pm 36$ & $292 \pm 34$ & 0.0003 \\
\hline Serum insulin iAUC (nmol/l) & $35 \pm 5$ & $38 \pm 6$ & 0.48 & $34 \pm 4$ & $40 \pm 8$ & 0.27 & $37 \pm 4$ & $43 \pm 8$ & 0.28 \\
\hline Insulin secretion iAUC $\left(\mathrm{nmol} \mathrm{min}{ }^{-1} \mathrm{~m}^{-2}\right)$ & $211 \pm 14$ & $201 \pm 22$ & 0.62 & $253 \pm 23$ & $252 \pm 42$ & 0.96 & $233 \pm 23$ & $219 \pm 28$ & 0.58 \\
\hline Insulin clearance $\left(1 \mathrm{~min}^{-1} \mathrm{~m}^{-2}\right)$ & $2.0 \pm 0.2$ & $1.8 \pm 0.1$ & 0.07 & $2.2 \pm 0.2$ & $2.0 \pm 0.2$ & 0.02 & $2.0 \pm 0.2$ & $1.8 \pm 0.2$ & 0.03 \\
\hline Insulin sensitivity $\left(\mathrm{ml} \mathrm{min} \operatorname{mos}^{-1} \mathrm{~m}^{-2}[\mathrm{pmol} / \mathrm{l}]^{-1}\right)$ & $0.11 \pm 0.02$ & $0.10 \pm 0.02$ & 0.69 & $0.05 \pm 0.01$ & $0.04 \pm 0.01$ & 0.41 & $0.02 \pm 0.01$ & $0.02 \pm 0.01$ & 0.81 \\
\hline Rate sensitivity $\left(\mathrm{pmol} \mathrm{m}{ }^{-2}[\mathrm{mmol} / \mathrm{l}]^{-1}\right.$ ) & $919 \pm 191$ & $1,452 \pm 278$ & 0.06 & $646 \pm 117$ & $800 \pm 135$ & 0.29 & $535 \pm 150$ & $327 \pm 122$ & 0.15 \\
\hline $\begin{array}{l}\text { Glucose sensitivity (pmol } \mathrm{min}^{-1} \mathrm{~m}^{-2} \\
{[\mathrm{mmol} / \mathrm{l}]^{-1} \text { ) }}\end{array}$ & $112 \pm 13$ & $135 \pm 21$ & 0.19 & $79 \pm 9$ & $93 \pm 11$ & 0.19 & $49 \pm 6$ & $86 \pm 10$ & 0.002 \\
\hline Potentiation factor ratio & $1.2 \pm 0.1$ & $1.4 \pm 0.1$ & 0.06 & $1.4 \pm 0.2$ & $1.9 \pm 0.2$ & 0.06 & $1.3 \pm 0.1$ & $1.6 \pm 0.2$ & 0.27 \\
\hline ISR@FPG (pmol min $\left.{ }^{-1} \mathrm{~m}^{-2}\right)$ & $95 \pm 17$ & $101 \pm 19$ & 0.66 & $93 \pm 13$ & $159 \pm 23$ & 0.003 & $148 \pm 12$ & $192 \pm 21$ & 0.01 \\
\hline $\mathrm{RaO}$ iAUC $\left(\mathrm{mmol} \mathrm{min} \operatorname{mog}^{-1}\left[\mathrm{~kg}_{\mathrm{FFM}}\right]^{-1}\right)$ & $3.9 \pm 0.3$ & $3.3 \pm 0.4$ & 0.09 & $3.7 \pm 0.3$ & $2.8 \pm 0.3$ & 0.003 & $2.7 \pm 0.2$ & $1.7 \pm 0.2$ & 0.03 \\
\hline $\mathrm{GCl}$ iAUC $\left(\mathrm{ml} \mathrm{min}^{-1}\left[\mathrm{~kg}_{\mathrm{FFM}}\right]^{-1}\right)$ & $277 \pm 41$ & $262 \pm 30$ & 0.72 & $138 \pm 32$ & $177 \pm 35$ & 0.42 & $27 \pm 40$ & $100 \pm 21$ & 0.20 \\
\hline Serum GLP-1 iAUC (pmol/1) & $-102 \pm 348$ & $755 \pm 377$ & 0.01 & $967 \pm 157$ & $1,699 \pm 234$ & 0.007 & $639 \pm 108$ & $1,531 \pm 270$ & 0.007 \\
\hline Serum GIP iAUC (nmol/l) & $6.1 \pm 1.0$ & $12.4 \pm 2.1$ & 0.0006 & $5.3 \pm 0.9$ & $13.2 \pm 2.4$ & 0.001 & $12.4 \pm 2.1$ & $23.6 \pm 2.6$ & 0.003 \\
\hline Serum glucagon iAUC (pmol/l) & $-697 \pm 144$ & $-106 \pm 257$ & 0.01 & $-206 \pm 110$ & $582 \pm 205$ & 0.01 & $-16 \pm 166$ & $741 \pm 279$ & 0.01 \\
\hline
\end{tabular}

Data are means \pm SEM

All iAUC were calculated by the trapezoidal rule as areas above baseline over $150 \mathrm{~min}$ (from -30 to $120 \mathrm{~min}$ )

The $p$ values were obtained from Student's paired $t$ test

FFM, fat-free mass; T2D, type 2 diabetes

response to the nutrient preload, when the volunteers were analysed altogether (ANOVA for repeated measures), we observed an enhancement in glucose sensitivity $(p<0.002)$, in potentiation $(p<0.02)$ and in ISR@FPG $(p<0.0001)$; however, differences attributable to glucose tolerance status emerged as statistically significant (interaction nutrients $\times$ group); namely, ISR@FPG increased only in participants with IGT and type 2 diabetes (Table 2), while glucose sensitivity increased by $20 \%$ in NGT and IGT groups, whereas it almost doubled in the type 2 diabetes group (Fig. 2).

Glucose fluxes In the 30 volunteers undergoing the double tracer protocol, the glycaemic responses of the two studies did not differ significantly from those of the whole population (Fig. 1 and electronic supplementary material [ESM] Fig. 1). In the water test, glucose absorption - as estimated by the $\mathrm{RaO}$ - was lower in type 2 diabetic individuals compared with those with NGT and IGT $(p<0.03$; Fig. 3 ). The nutrient preload caused a marked reduction of $\mathrm{RaO}$ that was more pronounced in patients with IGT and type 2 diabetes. As far as multiple regression analysis can estimate in this small number of participants, the delayed $\mathrm{RaO}$ (Standardised $\beta=0.42$ ) played a major role with respect to the improvement in beta cell function (Standardised $\beta=0.12$ ) in explaining the improved glucose tolerance. During the water OGTT, the EGP was suppressed in participants with NGT and IGT and only marginally affected in those with type 2 diabetes. The preload had no effect on basal EGP ( -30 to $0 \mathrm{~min}$ ) in any study group; after glucose ingestion, it marginally attenuated the physiological EGP decline in NGT, whereas it had no effect in patients with IGT and type 2 diabetes. Plasma $\mathrm{GCl}$ was stimulated by glucose ingestion and it followed, though with a delay, the plasma insulin concentration

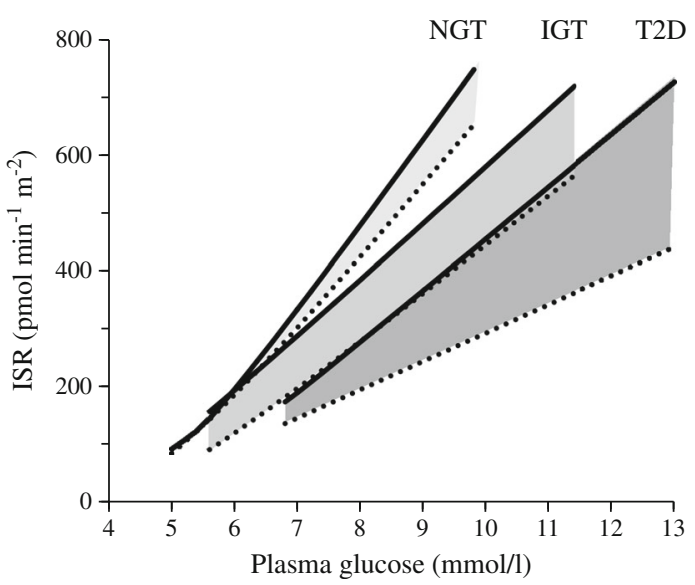

Fig. 2 Glucose-ISR dose-response curves during two $75 \mathrm{~g}$ OGTTs preceded at $-30 \mathrm{~min}$ by a preload of either water (dotted lines) or nutrients + water (black line) in NGT (light grey shading), IGT (middle grey shading) and type 2 diabetes (T2D; dark grey shading) groups 

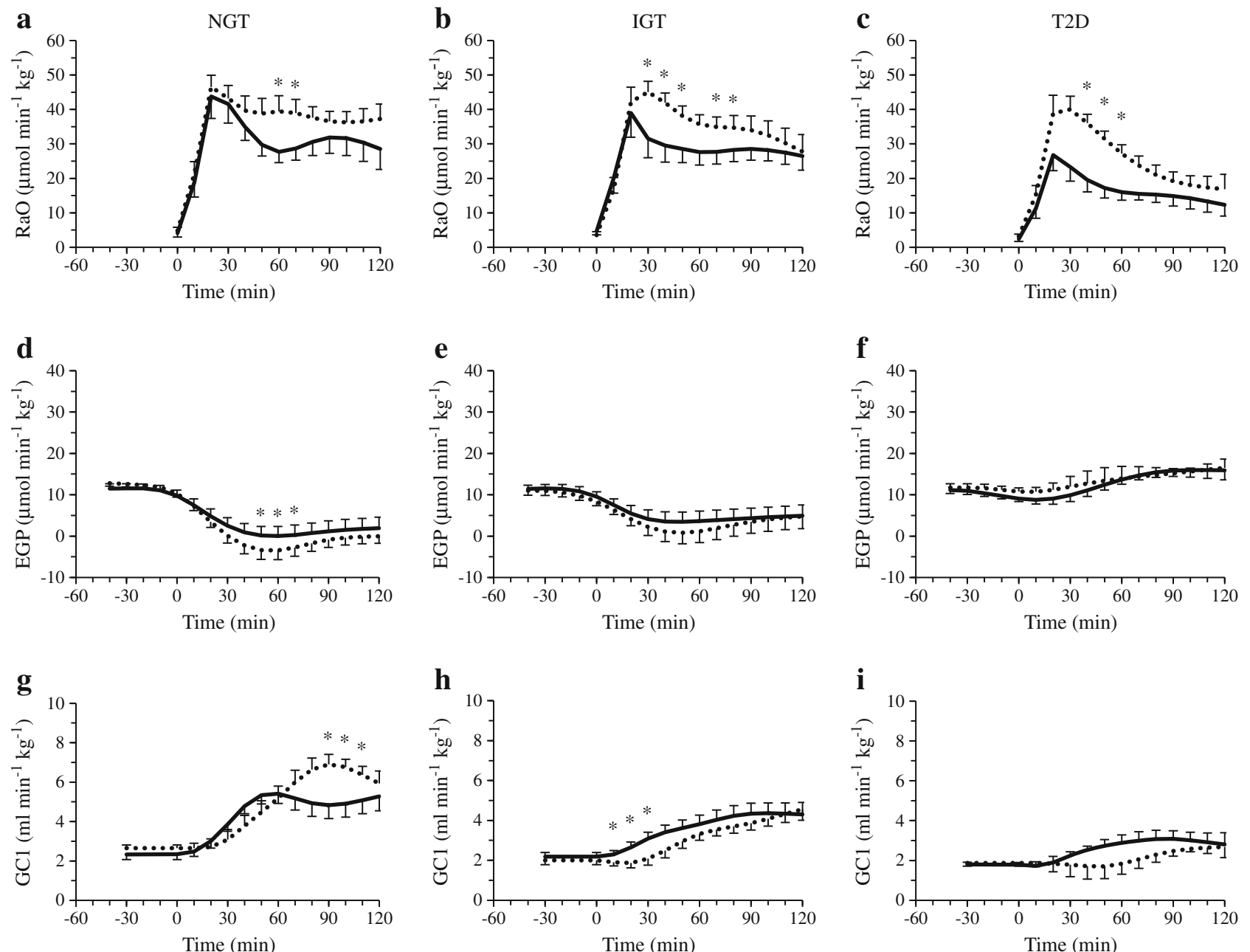

Fig. $3 \mathrm{RaO}(\mathbf{a}-\mathbf{c})$, EGP $(\mathbf{d}-\mathbf{f})$ and $\mathrm{GCl}(\mathbf{g}-\mathbf{i})$ of plasma glucose during two $75 \mathrm{~g}$ OGTTs preceded at $-30 \mathrm{~min}$ by a preload of either water (dotted lines) or nutrients + water (black line) in NGT $(\mathbf{a}, \mathbf{d}, \mathbf{g})$, IGT $(\mathbf{b}, \mathbf{e}, \mathbf{h})$ and

in the two tests, showing after the preload a slightly more prompt rise and, only in the NGT group, a delayed peak (Fig. 3).

GLP-1, GIP and glucagon Basal plasma GLP-1, GIP and glucagon levels were not statistically different on the two study days (Fig. 4). In response to the preload alone ( -30 to $0 \mathrm{~min}$ ), GIP showed a prompt small rise in all three groups, while no change was observed in either GLP-1 or glucagon concentrations. In response to glucose ingestion, in all groups, total GIP concentration rose promptly and remained elevated throughout the OGTT, total GLP-1 slightly increased and plasma glucagon declined smoothly. The preload almost doubled the glucose-induced GIP response, induced a modest rise in plasma GLP-1, particularly in the final part of the test, and prevented the decline in plasma glucagon concentration. All these effects were qualitatively similar in the three study groups, with a tendency to be more pronounced in IGT and type 2 diabetes groups with respect to NGT. No correlations type 2 diabetes (T2D; $\mathbf{c}, \mathbf{f}, \mathbf{i})$ groups. All indexes are standardised for individual fat-free mass. ${ }^{*} p<0.05$ water vs nutrients

were found between GLP-1, GIP and glucagon changes and changes in glucose fluxes and/or beta cell function variables.

PP Fasting PP concentrations were similar between the two studies and the two study groups in which they were measured (NGT and IGT). The nutrient preload stimulated a minor, not statistically significant increase of PP secretion. Glucose ingestion did not stimulate further PP release, neither in the water nor in the nutrients study (data not shown).

NEFA Fasting plasma NEFA were higher in participants with IGT and type 2 diabetes than in those with NGT $(p<0.02)$. In all groups, plasma NEFA remained stable after the preload and showed a progressive decline during the water OGTT study, which was influenced by the glucose tolerance status $(80 \%$ in NGT, $75 \%$ in IGT and $60 \%$ in type 2 diabetes) but not by the preload (data not shown). 

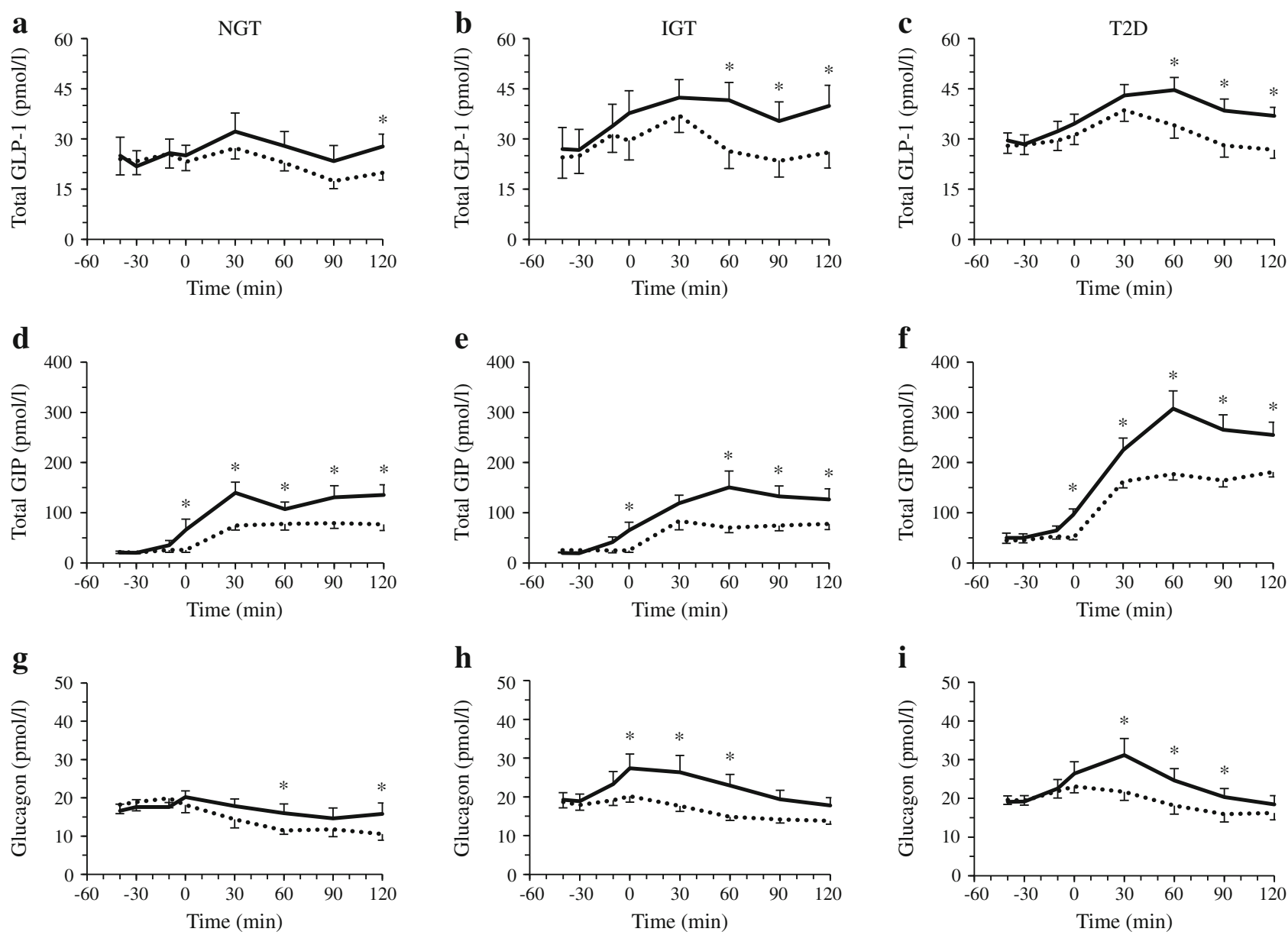

Fig. 4 Concentrations of plasma GLP-1 (a-c), GIP $(\mathbf{d}-\mathbf{f})$ and glucagon $(\mathbf{g}-\mathbf{i})$ during two $75 \mathrm{~g}$ OGTTs preceded at -30 min by a preload of either water (dotted lines) or nutrients + water (black line) in NGT (a,d,g), IGT (b,e,h) and type 2 diabetes (T2D; c,f,i) groups. * $p<0.05$ water vs nutrients

\section{Discussion}

The present study shows that a small lipid and protein preload significantly reduces the $2 \mathrm{~h}$ plasma glucose response to oral glucose ingestion and that the size of this effect is strongly dependent $\left(r^{2}=0.38, p>0.0001\right)$ on the degree of glucose tolerance impairment (Fig. 5). The underlying mechanism includes combinations of delayed glucose absorption (in all participants) and improvements in insulin secretion and clearance, which are quantitatively and qualitatively different in individuals with NGT, IGT and type 2 diabetes. The NGT group showed only mild changes (of borderline statistical significance) in insulin clearance, beta cell rate sensitivity and potentiation, while IGT and type 2 diabetes groups showed an enhancement of the beta cell baseline secretion (ISR@FPG) and a small but consistent reduction in insulin clearance that, only in patients with type 2 diabetes, were associated with a large improvement in beta cell glucose sensitivity. These effects were associated with - but not proportional to - an amplification of the physiological GLP-1 and GIP responses to glucose ingestion.

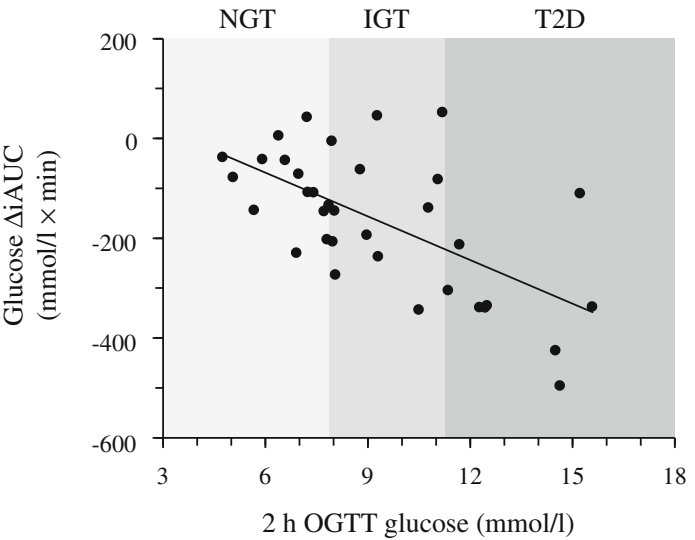

Fig. 5 Relationship between the individual glucose tolerance, as measured with the water test ( $2 \mathrm{~h}$ OGTT glucose), and the change in the plasma glucose iAUC (Glucose $\triangle \mathrm{iAUC}$ ) induced by the nutrient preload in NGT (light grey shading), IGT (middle grey shading) and type 2 diabetes (T2D; dark grey shading) groups. iAUCs were calculated by the trapezoidal rule as areas above baseline over $150 \mathrm{~min}$ (from -30 to $120 \mathrm{~min})$ 
In quantitative terms, the reduced $\mathrm{RaO}$ after the nutrient preload was the major determinant of the improved glucose tolerance. Physiologically, this effect could be explained by feedbacks arising from the interaction of nutrients with the stomach and/or with the small intestine, which are able to produce a delay in gastric emptying and a reduction in intestinal motility [35], either directly by altering the chemical and physical characteristics of the gastrointestinal milieu [11] or indirectly through neural activation and hormone release (e.g. GLP-1, peptide YY and cholecystokinin) [35]. Gastric emptying accounts for $\approx 35 \%$ of the variance in the glycaemic response to oral glucose in healthy and type 2 diabetic patients, while the extent to which a variation in small intestinal motility and absorptive function affects glucose tolerance remains uncertain $[5,35]$. The relationship between gastric emptying and glycaemia is complex, time-dependent and variable among different classes of glucose tolerance [36]. As we neither directly measured gastric emptying nor small intestinal motility, we cannot dissect the contribution of each component to the rate of glucose absorption. Nevertheless, the reduction of $\mathrm{RaO}$ after the nutrient preload was progressively more pronounced across glucose tolerance status $(-15,-25$ and $-37 \%$, respectively in NGT, IGT and type 2 diabetes groups), therefore this implies that the mechanisms referred above are at least preserved and/or have a progressively greater impact in the transition from normal to abnormal glucose homeostasis, owing to concomitant changes in insulin secretion dynamics and insulin sensitivity.

The delay in glucose absorption might be caused by the preload-induced release of GLP-1, whose effect on gastric emptying has been reported to be dose-dependent and substantially preserved in type 2 diabetes [37]. In our study, although GLP-1 plasma concentration had a tendency to be higher in individuals with IGT and type 2 diabetes and was further increased by nutrient ingestion, we could not find any correlations between changes in plasma GLP-1 and RaO. Cholecystokinin and peptide YY could be involved since they also modulate gastrointestinal motility in response to nutrient ingestion; however, it is unlikely that they can explain the large differences in the slow-down of glucose absorption observed among the three study groups.

With regard to beta cell function, interestingly, in our diabetic patients the beta cell glucose sensitivity, though markedly impaired ( $40 \%$ the value of NGT), was still very responsive to the physiological modulation induced by nutrient ingestion, displaying almost a full recovery (Fig. 2). GLP-1 and GIP, the two hormones possibly involved in this 'incretin effect' [38], displayed a different response. Plasma GLP-1 was only marginally affected by the nutrient preload (probably due to its low caloric and glycaemic content [37]) and after the ingestion of glucose it rose similarly in patients with IGT and type 2 diabetes (to values also reported by other laboratories [1, 2]). GIP release, in agreement with other studies [38], was stimulated by both nutrients and glucose ingestion, reaching the highest values in type 2 diabetes patients. In our study, we could not find any significant association between GLP-1 or GIP plasma concentrations (or changes) and changes in beta cell function; however, it must be taken into account that the insulinotropic effects of both GLP-1 and GIP-although negatively affected by obesity and diabetes [38] — are dependent on glucose concentration [38] and expected to be more evident in hyperglycaemic patients. The activation of the parasympathetic nervous system $[39,40]$ and the rise of specific plasma substrates derived from nutrient digestion $[9,41]$ might also play a role. The cephalic component of insulin secretion, defined as the insulin release occurring within minutes after food sensory stimulation [7], might have been missed by our study; however, it is transient $(10 \mathrm{~min})$ and modest $(<20 \mathrm{pmol} / 1$ of insulin) therefore, at least in our experimental condition, it is unlikely to be relevant. The possibility that the parasympathetic system is more activated is also unlikely on the basis of others' [7] as well as our data on plasma PP, showing small changes and a wide interindividual variability. The rise in specific plasma substrates after nutrients digestion may also stimulate insulin release. In the present study, we can exclude plasma NEFA concentrations, which were not influenced by the nutrient preload, but we cannot exclude that changes in plasma amino acid concentrations may have directly promoted insulin secretion [42]. If confirmed, this effect might help to explain why beta cell glucose sensitivity was improved particularly in type 2 diabetic patients, as the plasma amino acid rise after a mixed meal was actually increased in patients with type 2 diabetes as compared with age- and weight-matched non-diabetic controls [43] and the sensitivity of beta cells to amino acid stimulation is preserved in this condition [44].

The nutrient preload also induced a $10 \%$ reduction of insulin clearance, which is in full agreement with what has been previously reported following a $30 \mathrm{~g}$ protein preload [6]. This reduction - though consistent-is small and therefore unlikely to significantly contribute to the improvement in glucose tolerance. Indeed, it could depend on an increased renal C-peptide clearance due to the amino acid-induced increment of glomerular filtration rate. If this were really the case, we would have underestimated the improvement in insulin secretion, as it is based on modelling C-peptide plasma kinetics assuming a constant clearance [30].

Overall, the current results suggest that exploiting nutrient sensing (i.e. the physiological systems that are activated by nutrient ingestion, directly or by neuro-hormonal signals) could be an effective and attractive approach to treat postprandial hyperglycaemia. In terms of effect size, our protein/lipid preload is similar or superior to what can be achieved with the 
available anti-hyperglycaemic drugs [45]. Whether the nutrient preload has an impact on total daily energy intake and its effect persists long-term can only be assessed by an ad hoc designed randomised clinical trial; however, it has recently been suggested that the effect of a $25 \mathrm{~g}$ whey preload is persistent and neutral on body weight [46]. The preload we used may not be optimal for the timing ( $30 \mathrm{~min}$ before the glucose challenge), the caloric content and the lipid quality; it was chosen as an investigational tool in keeping with previous studies from our laboratory [47]. In addition, our data show that the improvement in glucose tolerance occurred despite the stimulation of glucagon release (a consequence of protein digestion) that was associated with a less effective EGP suppression. Therefore, we can speculate that adding a small amount of carbohydrate to the preload would improve its hypoglycaemic power by engaging the 'Staub-Traugott effect', i.e., the potentiation of EGP suppression that occurs when a glucose load is preceded by glucose ingestion [48].

The short duration of the post-absorptive phase of the study ( $2 \mathrm{~h}$ ) represents a limitation since we do not know whether the glycaemic excursion - although milder - would last longer in the patients and, ideally, the evaluation of each main outcome would have been more robust if plasma glucose had reached the baseline values.

In conclusion, a small protein and lipid preload is able to slow-down glucose absorption and improve beta cell function to an extent that is proportional to the deterioration of glucose tolerance. Exploiting the physiological mechanisms activated by nutrient sensing might reveal an interesting tool in the treatment and/or prevention of type 2 diabetes.

Acknowledgements We would like to thank the volunteers enrolled in this trial and S. Burchielli (Department of Clinical and Experimental Medicine, University of Pisa, Pisa, Italy) for secretarial assistance.

Funding This work was supported by grants from the University of Pisa (Fondi di Ateneo) and by FCT grant (PIC/IC/82956/2007).

Duality of interest The authors declare that there is no duality of interest associated with this manuscript.

Author contributions DT conducted the clinical studies, provided a substantial contribution to the acquisition, analysis and interpretation of the data and drafted the manuscript. SB and SF provided a substantial contribution to the data collection and analysis. AT provided a substantial contribution to the data collection and interpretation. MPM provided a substantial contribution to the conception and design of the experiments and the interpretation of the data. AM provided a substantial contribution to the generation and interpretation of the tracer data. EF provided a substantial contribution to the interpretation of the data. AN conceived and designed the study, provided a substantial contribution to the analysis and interpretation of the data, and revised the manuscript. All authors revised critically the manuscript and approved the final version of the article. DT and AN are responsible for the integrity of the data and the accuracy of the data analysis.

\section{References}

1. Gentilcore D, Chaikomin R, Jones KL et al (2006) Effects of fat on gastric emptying of and the glycemic, insulin, and incretin responses to a carbohydrate meal in type 2 diabetes. J Clin Endocrinol Metab 91:2062-2067

2. Ma J, Stevens JE, Cukier K et al (2009) Effects of a protein preload on gastric emptying, glycemia, and gut hormones after a carbohydrate meal in diet-controlled type 2 diabetes. Diabetes Care 32: $1600-1602$

3. Gannon MC, Nuttall FQ (2004) Effect of a high-protein, lowcarbohydrate diet on blood glucose control in people with type 2 diabetes. Diabetes 53:2375-2382

4. O'Connor LM, Lentjes MA, Luben RN, Khaw KT, Wareham NJ, Forouhi NG (2014) Dietary dairy product intake and incident type 2 diabetes: a prospective study using dietary data from a 7-day food diary. Diabetologia 57:909-917

5. Marathe CS, Rayner CK, Jones KL, Horowitz M (2013) Relationships between gastric emptying, postprandial glycemia, and incretin hormones. Diabetes Care 36:1396-1405

6. Lan-Pidhainy X, Wolever TM (2010) The hypoglycemic effect of fat and protein is not attenuated by insulin resistance. Am J Clin Nutr 91:98-105

7. Teff KL (2011) How neural mediation of anticipatory and compensatory insulin release helps us tolerate food. Physiol Behav 103:44-50

8. Carr RD, Larsen MO, Winzell MS et al (2008) Incretin and islet hormonal responses to fat and protein ingestion in healthy men. Am J Physiol Endocrinol Metab 295:E779-784

9. Newsholme P, Cruzat V, Arfuso F, Keane K (2014) Nutrient regulation of insulin secretion and action. J Endocrinol 221:R105-120

10. Collins PJ, Horowitz M, Cook DJ, Harding PE, Shearman DJ (1983) Gastric emptying in normal subjects-a reproducible technique using a single scintillation camera and computer system. Gut 24:1117-1125

11. Kong F, Singh RP (2008) Disintegration of solid foods in human stomach. J Food Sci 73:R67-80

12. Nuttall FQ, Gannon MC (1991) Plasma glucose and insulin response to macronutrients in nondiabetic and NIDDM subjects. Diabetes Care 14:824-838

13. Gannon MC, Nuttall FQ, Westphal SA, Seaquist ER (1993) The effect of fat and carbohydrate on plasma glucose, insulin, C-peptide, and triglycerides in normal male subjects. J Am Coll Nutr 12:36-41

14. Moghaddam E, Vogt JA, Wolever TM (2006) The effects of fat and protein on glycemic responses in nondiabetic humans vary with waist circumference, fasting plasma insulin, and dietary fiber intake. J Nutr 136:2506-2511

15. Collier G, O'Dea K (1983) The effect of coingestion of fat on the glucose, insulin, and gastric inhibitory polypeptide responses to carbohydrate and protein. Am J Clin Nutr 37:941-944

16. Cunningham KM, Read NW (1989) The effect of incorporating fat into different components of a meal on gastric emptying and postprandial blood glucose and insulin responses. Br J Nutr 61:285-290

17. Houghton LA, Mangnall YF, Read NW (1990) Effect of incorporating fat into a liquid test meal on the relation between intragastric distribution and gastric emptying in human volunteers. Gut 31 : 1226-1229

18. Gannon MC, Ercan N, Westphal SA, Nuttall FQ (1993) Effect of added fat on plasma glucose and insulin response to ingested potato in individuals with NIDDM. Diabetes Care 16:874-880

19. Randle PJ, Garland PB, Hales CN, Newsholme EA (1963) The glucose fatty-acid cycle. Its role in insulin sensitivity and the metabolic disturbances of diabetes mellitus. Lancet 1:785-789

20. Karamanlis A, Chaikomin R, Doran S et al (2007) Effects of protein on glycemic and incretin responses and gastric emptying after oral glucose in healthy subjects. Am J Clin Nutr 86:1364-1368 
21. Gannon MC, Nuttall FQ, Neil BJ, Westphal SA (1988) The insulin and glucose responses to meals of glucose plus various proteins in type II diabetic subjects. Metab Clin Exp 37:1081-1088

22. Pilichiewicz A, O'Donovan D, Feinle C et al (2003) Effect of lipase inhibition on gastric emptying of, and the glycemic and incretin responses to, an oil/aqueous drink in type 2 diabetes mellitus. J Clin Endocrinol Metab 88:3829-3834

23. O'Donovan D, Horowitz M, Russo A et al (2004) Effects of lipase inhibition on gastric emptying of, and on the glycaemic, insulin and cardiovascular responses to, a high-fat/carbohydrate meal in type 2 diabetes. Diabetologia 47:2208-2214

24. Bock G, Dalla Man C, Campioni M et al (2007) Effects of nonglucose nutrients on insulin secretion and action in people with pre-diabetes. Diabetes 56:1113-1119

25. American Diabetes Association (2014) Standards of medical care in diabetes-2014. Diabetes Care 37(Suppl 1):S14-S80

26. Kwiatek MA, Menne D, Steingoetter A et al (2009) Effect of meal volume and calorie load on postprandial gastric function and emptying: studies under physiological conditions by combined fiberoptic pressure measurement and MRI. Am J Physiol Gastrointest Liver Physiol 297:G894-901

27. Jakubowicz D, Froy O, Ahren B et al (2014) Incretin, insulinotropic and glucose-lowering effects of whey protein pre-load in type 2 diabetes: a randomised clinical trial. Diabetologia 57:1807-1811

28. Gastaldelli A, Casolaro A, Pettiti M et al (2007) Effect of pioglitazone on the metabolic and hormonal response to a mixed meal in type II diabetes. Clin Pharmacol Ther 81:205-212

29. Mari A, Stojanovska L, Proietto J, Thorburn AW (2003) A circulatory model for calculating non-steady-state glucose fluxes. Validation and comparison with compartmental models. Comput Methods Prog Biomed 71:269-281

30. Van Cauter E, Mestrez F, Sturis J, Polonsky KS (1992) Estimation of insulin secretion rates from C-peptide levels. Comparison of individual and standard kinetic parameters for C-peptide clearance. Diabetes 41:368-377

31. Mari A, Ferrannini E (2008) Beta-cell function assessment from modelling of oral tests: an effective approach. Diabetes Obes Metab 10(Suppl 4):S77-S87

32. Mari A, Schmitz O, Gastaldelli A, Oestergaard T, Nyholm B, Ferrannini E (2002) Meal and oral glucose tests for assessment of beta-cell function: modeling analysis in normal subjects. Am J Physiol Endocrinol Metab 283:E1159-1166

33. Mari A, Tura A, Gastaldelli A, Ferrannini E (2002) Assessing insulin secretion by modeling in multiple-meal tests: role of potentiation. Diabetes 51(Suppl 1):S221-S226
34. Jimenez A, Mari A, Casamitjana R, Lacy A, Ferrannini E, Vidal J (2014) GLP-1 and glucose tolerance after sleeve gastrectomy in morbidly obese subjects with type 2 diabetes. Diabetes 63:33723377

35. Chaikomin R, Rayner CK, Jones KL, Horowitz M (2006) Upper gastrointestinal function and glycemic control in diabetes mellitus. World J Gastroenterol 12:5611-5621

36. Trahair LG, Horowitz M, Marathe CS, et al (2014) Impact of gastric emptying to the glycemic and insulinemic responses to a 75-g oral glucose load in older subjects with normal and impaired glucose tolerance. Physiol Rep 2:c12204

37. Meier JJ, Gallwitz B, Salmen S et al (2003) Normalization of glucose concentrations and deceleration of gastric emptying after solid meals during intravenous glucagon-like peptide 1 in patients with type 2 diabetes. J Clin Endocrinol Metab 88:2719-2725

38. Nauck MA, Baller B, Meier JJ (2004) Gastric inhibitory polypeptide and glucagon-like peptide- 1 in the pathogenesis of type 2 diabetes. Diabetes 53(Suppl 3):S190-S196

39. Teff K (2000) Nutritional implications of the cephalic-phase reflexes: endocrine responses. Appetite 34:206-213

40. Ahren B (2000) Autonomic regulation of islet hormone secretionimplications for health and disease. Diabetologia 43:393-410

41. Nolan CJ, Prentki M (2008) The islet beta-cell: fuel responsive and vulnerable. Trends Endocrinol Metab 19:285-291

42. Gannon MC, Nuttall FQ (2010) Amino acid ingestion and glucose metabolism-a review. IUBMB Life 62:660-668

43. Muscelli E, Frascerra S, Casolaro A et al (2014) The amino acid response to a mixed meal in patients with type 2 diabetes: effect of sitagliptin treatment. Diabetes Obes Metab 16:1140-1147

44. Floyd JC Jr, Fajans SS, Conn JW, Thiffault C, Knopf RF, Guntsche E (1968) Secretion of insulin induced by amino acids and glucose in diabetes mellitus. J Clin Endocrinol Metab 28:266-276

45. Stein SA, Lamos EM, Davis SN (2013) A review of the efficacy and safety of oral antidiabetic drugs. Expert Opin Drug Saf 12:153175

46. Ma J, Jesudason DR, Stevens JE et al (2015) Sustained effects of a protein 'preload' on glycaemia and gastric emptying over 4 weeks in patients with type 2 diabetes: a randomized clinical trial. Diabetes Res Clin Pract 108:e31-34

47. Camastra S, Muscelli E, Gastaldelli A et al (2013) Long-term effects of bariatric surgery on meal disposal and beta-cell function in diabetic and nondiabetic patients. Diabetes 62:3709-3717

48. Bonuccelli S, Muscelli E, Gastaldelli A et al (2009) Improved tolerance to sequential glucose loading (Staub-Traugott effect): size and mechanisms. Am J Physiol Endocrinol Metab 297:E532-537 